\title{
Numerical Solution of Obstacle Problems by B-Spline Functions
}

\author{
Ghasem Barid Loghmani ${ }^{1}$, Farshad Mahdifar ${ }^{2}$, Seyed Rouhollah Alavizadeh ${ }^{1}$ \\ ${ }^{1}$ Department of Mathematics, Yazd University, Yazd, Iran \\ ${ }^{2}$ Department of Mathematics, Payame Noor University, Tehran, Iran \\ E-mail: loghmani@yazduni.ac.ir,farshad.mehdifar@pnu.ac.ir \\ Received February 19, 2011; revised April 6, 2011; accepted April 15, 2011
}

\begin{abstract}
In this study, we use B-spline functions to solve the linear and nonlinear special systems of differential equations associated with the category of obstacle, unilateral, and contact problems. The problem can easily convert to an optimal control problem. Then a convergent approximate solution is constructed such that the exact boundary conditions are satisfied. The numerical examples and computational results illustrate and guarantee a higher accuracy for this technique.
\end{abstract}

Keywords: Least Square Method, Uniform B-Splines, Boundary Value Problems, Obstacle Problems

\section{Introduction}

Variational inequality theory has become an effective and powerful tool for studying obstacle and unilateral problems arising in mathematical and engineering sciences. This theory has developed into an interesting branch of applicable mathematics, which contains a wealth of new ideas for inspiration and motivation to do research. It has been shown by Kikuchi and Oden [1] that the problem of equilibrium of elastic bodies in contact with a right foundation can be studied in the framework of variational inequality theory. Various numerical methods are being developed and applied to find the numerical solutions of the obstacle problems including finite difference techniques and spline based methods. In principle, these methods cannot be applied directly to solve the obstacle problems. However, if the obstacle function is known, one can characterize the obstacle problem by a sequence of boundary value problems without constraints via the variational inequality and a penalty function. The computational advantage of this approach is its simple applicability for solving differential equations. Such type of penalty function methods have been used quite effectively by Noor and Tirmizi [2], as a basis for obtaining numerical solutions for some obstacle problems.

The aim of this paper is to consider the use of quadratic B-spline functions and least square method to develop a numerical method for obtaining smooth appro- ximations for the solution and its derivatives of the general form of a system of second order boundary value problem of the type.

$$
\begin{gathered}
u^{\prime \prime}(t)=\left\{\begin{array}{l}
g_{1}\left(t, u(t), u^{\prime}(t)\right), \quad a=a_{1} \leq t \leq a_{2} \\
g_{2}\left(t, u(t), u^{\prime}(t)\right), \quad a_{2} \leq t \leq a_{3} \\
g_{3}\left(t, u(t), u^{\prime}(t)\right), \quad a_{3} \leq t \leq a_{4}=b
\end{array}\right. \\
u(a)=\alpha_{1} \text { and } u(b)=\alpha_{2},
\end{gathered}
$$

and the continuity conditions of $u$ and $u^{\prime}$ at $a_{2}$ and $a_{3}$. Here, $g_{i}:\left[a_{i}, b_{i+1}\right] \times \mathrm{R}^{2} \rightarrow \mathrm{R} \quad(i=1,2,3)$, are given continuous functions, and the parameters $\alpha_{1}$ and $\alpha_{2}$ are real finite constants. Linear form of such type of systems arise in the study of one dimensional obstacle, unilateral, moving and free boundary value problems, [1,3-10] and the references therein. In general, it is not possible to obtain the analytic form of the solution of (1)-(2) for arbitary choices of $g_{i}\left(t, u, u^{\prime}\right),(i=1,2$, 3 ), so we resort to some numerical methods for obtaining an approximate solution of (1)-(2).

In 1981, Villaggio [11] used the classical RayleighRitz method for solving a special form of (1), namely,

$$
\begin{gathered}
u^{\prime \prime}(t)=\left\{\begin{array}{c}
0, \quad 0 \leq t \leq \frac{\pi}{4} \text { and } \frac{3 \pi}{4} \leq t \leq \pi \\
u(t)-1, \quad \frac{\pi}{4} \leq t \leq \frac{3 \pi}{4}
\end{array}\right. \\
u(0)=0 \text { and } u(\pi)=0,
\end{gathered}
$$

and the continuity conditions of $u$ and $u^{\prime}$ at $\frac{\pi}{4}$ and 
$\frac{3 \pi}{4}$. Later, Noor and Khalifa [12] have solved problem (3)-(4) using collocation method with cubic splines as basis functions. Similar conclusions were pointed out by Noor and Tirmizi [2], Al-Said [13] and Al-Said et al. [14], where second and fourth order finite difference and spline methods were used to solve a special linear form of problem (1), namely,

$$
\begin{gathered}
u^{\prime \prime}(t)=\left\{\begin{array}{c}
f(t), \\
a \leq t \leq a_{2} \text { and } a_{3} \leq t \leq b \\
g(t) u(t)+f(t)+r, \\
a_{2} \leq t \leq a_{3}
\end{array}\right. \\
u(a)=\alpha_{1} \text { and } u(b)=\alpha_{2} .
\end{gathered}
$$

where, the functions $f(t)$ and $g(t)$ are continuous on [a, b] and $\left[a_{2}, a_{3}\right]$, respectively and the continuity conditions of $u$ and $u^{\prime}$ at $a_{2}$ and $a_{3}$ is assumed, and $r$ is a real finite constants parameter. On the other hand, Al-Said [3,15,16] has developed and analyzed quadratic and cubic spline methods for solving (5)-(6) and compared his numerical results with other available results given in [2,12]. It was shown in [16] that the cubic spline method gives much better results than those produced by other methods (including the fourth order Nemerov method).

In 2003, Khan and Aziz [17] have solved problem (1)-(2) using parametric cubic spline technique and have shown that the their method gives approximations which are better than that produced by Al-Said method [16]. Then in 2005, Siraj-ul-Islam, Aslam Noor, Tirmizi and Azam Khan [18] have established and analyzed optimal smooth approximations for systems of second order boundary value problems of the form (5)-(6) with quadratic non-polynomial splines. Also in 2006, similar methods were pointed out by Siraj-ul-Islam and Tirmizi [19] who developed a class of methods based on cubic non-polynomial splines for problem (5)-(6). The obtained results in $[18,19]$ are very encouraging and non-polynomial spline methods perform better than other existing methods [2,3,12-17] of the same order. Owing to importance of problem (5)-(6) in physics, the existence and uniqueness of solution to this problems has been related with one dimensional second order obstacle boundary value problems. Moreover, existence and uniqueness theorem for obstacle problems has been studied by variational inequalities theory and demonstrate by Friedman [5] and also by Kinderlehrer and Stampacchia [7], see, for more examples $[1,6,10]$. But in general form (1)-(2), it has been no very discussion.

In this paper, we shall solve the general problem numerically (1)-(2) by scaling functions $\Phi_{k, i}(t)$ for $k \in \mathrm{N}$ and $\left(i=-2,-1,0, \cdots, 3 \cdot 2^{k-1}-1\right)$. Our presentation finds a sequence of functions $\left\{v_{k}\right\}$ of the form

$$
v_{k}(t)=\sum_{i=-2}^{3 \cdot 2^{k-1}-1} c_{i} \Phi_{k, i}(t),
$$

which satisfy the exact boundary conditions. Also, up to an error $\varepsilon_{k}$, the function $v_{k}$ satisfies the differential equation, where $\varepsilon_{k} \rightarrow 0$ as $k \rightarrow \infty$.

\section{Statement of the Method}

Consider, the general system of differential equations of the type

$$
u^{\prime \prime}(t)=\left\{\begin{array}{lc}
G_{1}[u(t)], & a=a_{1} \leq t \leq a_{2} \\
G_{2}[u(t)], & a_{2} \leq t \leq a_{3} \\
G_{3}[u(t)], & a_{3} \leq t \leq a_{4}=b
\end{array}\right.
$$

with the general boundary conditions

$$
U_{i}[u(t)]=\alpha_{i}, \quad i=1,2
$$

and the continuity conditions of $u$ and $u^{\prime}$ at $a_{2}$ and $a_{3}$, where $G_{i}$ and $U_{i}$ are second-order linear and boundary operators, respectively, and $U_{i}$ 's are operators defined in the forms

$$
U_{i}[u(t)]=\sum_{j=1}^{2} \lambda_{i j} u^{j-1}(a)+\sum_{j=1}^{2} \mu_{i j} u^{j-1}(b), \quad i=1,2
$$

where, $\lambda_{i j}, \mu_{i j}$ and $\alpha_{i}$ are real constants. Since, least square mehtod for system of the differential Equations (7)-(8) lead to complicated large scale and can not ensure existence and uniqueness of solution to this problems. Therefore, we will be study the system of two-point second-order boundary value problems of the type

$$
\begin{gathered}
u^{\prime \prime}(t)=\left\{\begin{array}{l}
g_{1}\left(t, u(t), u^{\prime}(t)\right), \quad a=a_{1} \leq t \leq a_{2} \\
g_{2}\left(t, u(t), u^{\prime}(t)\right), \quad a_{2} \leq t \leq a_{3} \\
g_{3}\left(t, u(t), u^{\prime}(t)\right), \quad a_{3} \leq t \leq a_{4}=b
\end{array}\right. \\
u(a)=\alpha_{1} \text { and } u(b)=\alpha_{2},
\end{gathered}
$$

and the continuity conditions of $u$ and $u^{\prime}$ at $a_{2}$ and $a_{3}$. Let $g_{i}:\left[a_{i}, b_{i+1}\right] \times \mathrm{R}^{2} \rightarrow \mathrm{R} \quad(i=1,2,3)$ be continuous functions. We convert the problem to an optimal control problem

$$
\min _{u} \sum_{i=1}^{3} \int_{a_{i}}^{a_{i}+1}\left(u^{\prime \prime}(t)-g_{i}\left(t, u(t), u^{\prime}(t)\right)\right)^{2} d t
$$

and two-point boundary conditions

$$
u(a)=\alpha_{1} \text { and } u(b)=\alpha_{2} .
$$

The actual solution of (1)-(2) is a function $v$ such that

$$
\left\{\begin{array}{c}
\sum_{i=1}^{3}\left\|v^{\prime \prime}(t)-g_{i}\left(t, v(t), v^{\prime}(t)\right)\right\|_{L^{2}\left(\left[a_{i}, a_{i+1}\right]\right)}^{2}=0 \\
v(a)=\alpha_{1}, v(b)=\alpha_{2}
\end{array} .\right.
$$


For all $\varepsilon>0$, the method finds an approximate solution $v_{\varepsilon}$ satisfying

$$
\left\{\begin{array}{l}
\sum_{i=1}^{3}\left\|v_{\varepsilon}^{\prime \prime}(t)-g_{i}\left(t, v_{\varepsilon}(t), v_{\varepsilon}^{\prime}(t)\right)\right\|_{L^{2}\left(\left[a_{i}, a_{i+1}\right]\right)}^{2}<\varepsilon \\
v_{\varepsilon}(a)=\alpha_{1}, v_{\varepsilon}(b)=\alpha_{2} .
\end{array}\right.
$$

The sketch of the method is delineated as follows:

Consider uniform quadratic B-spline function [20,21]

(Figure 1).

$$
B_{2}(t)=\frac{1}{2}\left\{\begin{array}{cc}
t^{2} & 0 \leq t<1 \\
-3(2-t)^{2}+(3-t)^{2} & 1 \leq t<2 \\
(3-t)^{2} & 2 \leq t<3 \\
0 & \text { otherwise }
\end{array}\right.
$$

For simplicity, the break up point of the interval [ $a$, $b$ ] are taken at $a_{2}=\frac{3 a+b}{4}$ and $a_{3}=\frac{3 a+b}{4}$ to develop the numerical method for approximating solution of a system of differential Equations (1)-(2). For a fix natural number $k \geq 3$, we divided the interval [ $a$, $b$ ] into $\left(3 \cdot 2^{k-1}\right)$ equal subinterval using the control points,

$$
\begin{gathered}
t_{i}=a+(i+2) h, t_{-2}=a, t_{3 \cdot 2^{k-1}-1}=b, \\
\left(i=-2,-1, \cdots, 3 \cdot 2^{k-1}-1\right),
\end{gathered}
$$

where $h=\frac{b-a}{3 \cdot 2^{k-1}}$, (with attention to grid points,

$$
\left.t_{3 \cdot 2^{k-3}-2}=a_{2}, t_{3^{2} \cdot 2^{k-3}-2}=a_{3}\right) \text {. }
$$

We define,

$$
\begin{gathered}
\Phi_{k, i}(t)=B_{2}\left(\frac{3 \cdot 2^{k-1}}{b-a}(t-a)-i\right), \\
\left(i=-2,-1, \cdots, 3 \cdot 2^{k-1}-1\right)
\end{gathered}
$$

where $B_{2}$ is a scaling function and $\Phi_{k, i}(k \geq 3$, $i=-2,-1, \cdots, 3 \cdot 2^{k-1}-1$ ) are translations and dilations of $B_{2}$ as prescribed in [22-26].

Let

$$
v_{k}(t)=\sum_{i=-2}^{3 \cdot 2^{k-1}-1} c_{i} \Phi_{k, i}(t),
$$

where the coefficients $\left\{c_{i}\right\}$ are determined from the conditions

$$
v_{k}(a)=\alpha_{1}, \quad v_{k}(b)=\alpha_{2},
$$

and the following least square problem:

$$
\min _{c_{i}} \sum_{i=1}^{3}\left\|v_{k}^{\prime \prime}(t)-g_{i}\left(t, v_{k}(t), v_{k}^{\prime}(t)\right)\right\|_{L^{2}\left(\left[a_{i}, a_{i+1}\right]\right)}^{2}
$$

The minimization problem is equivalent to the following nonlinear system:

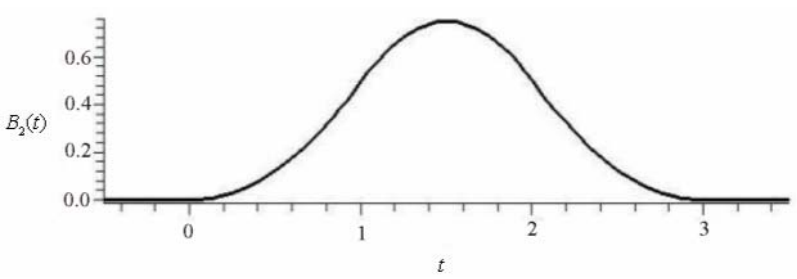

Figure 1. Uniform quadratic B-spline function.

$$
\left\{\begin{array}{c}
\sum_{i=1}^{3} \frac{\partial}{\partial c_{i}}\left\|v_{k}^{\prime \prime}(t)-g_{i}\left(t, v_{k}(t), v_{k}^{\prime}(t)\right)\right\|_{L^{2}\left(\left[a_{i}, a_{i+1}\right]\right)}^{2}=0, \\
\left(i=-2,-1, \cdots, 3 \cdot 2^{k-1}-1\right) \\
v_{k}(a)=\alpha_{1}, v_{k}(b)=\alpha_{2} .
\end{array}\right.
$$

\section{Convergence Analysis}

In this section, we analyzed new method in the special case of system of one-order boundary value problems of the type (1) with boundary condition $u(a)=\alpha_{1}$. However, consider the optimal control problem

$$
\min _{u} \int_{a}^{b} f\left(t, u(t), u^{\prime}(t)\right) d t
$$

and $u$ satisfying the boundary condition

$$
u\left(a_{1}\right)=\alpha_{1}
$$

where $a_{1}=a$. We supposes $f$ is in the form

$$
f\left(t, u(t), u^{\prime}(t)\right)=\left\{\begin{array}{c}
f_{1}\left(t, u(t), u^{\prime}(t)\right), \\
a=a_{1} \leq t \leq a_{2} \\
f_{2}\left(t, u(t), u^{\prime}(t)\right), \\
a_{2} \leq t \leq a_{3} \\
f_{3}\left(t, u(t), u^{\prime}(t)\right), \\
a_{3} \leq t \leq a_{4}=b
\end{array}\right.
$$

$$
f_{i}\left(t, u(t), u^{\prime}(t)\right):=\left[u^{\prime}(t)-g_{i}(t, u(t))\right]^{2}, \quad 1 \leq i \leq 3 .
$$

Consider the uniform linear B-spline function $[20,27$, 28] (Figure 2),

$$
B_{1}(t)= \begin{cases}t, & 0 \leq t<1 \\ 2-t, & 1 \leq t<2 \\ 0 & \text { otherwise. }\end{cases}
$$

For simplicity, suppose $a_{2}=\frac{3 a+b}{4}$ and $a_{3}=\frac{3 a+b}{4}$ such that divided the interval $[a, b]$ into $\left(2^{k}\right)$ equal subinterval using the control points,

$t_{i}=a+(i+1) h, t_{-1}=a, t_{2^{k}-1}=b,\left(i=-1, \cdots, 2^{k}-1\right)$, where $h=\frac{b-a}{2^{k}}$ and $k \geq 2$, (with attention to grid 


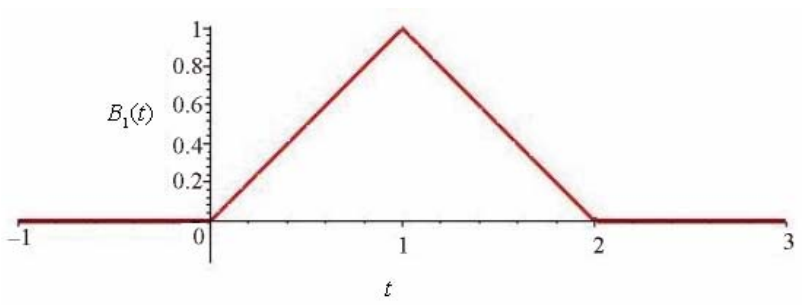

Figure 2. Uniform linear B-spline function (hat function).

points, $\left.t_{2^{k-2}-1}=a_{2}, t_{3 \cdot 2^{k-2}-1}=a_{3}\right)$.

Let $u(t)=\sum_{i=-1}^{2^{k}-1} c_{i} \varphi_{k, i}(t)$, where $\varphi_{k, i}(t):=B_{1}$

$\left(\frac{2^{k}}{b-a}(t-a)-i\right), i=-1,0,1,2, \cdots, 2^{k}-1$. $\left(\varphi_{k, i}\right.$ 's are translations and dilations of linear spline (hat function) $B_{1}$.

Then

$$
\begin{gathered}
\int_{a}^{b} f\left(t, u(t), u^{\prime}(t)\right) d t= \\
\int_{a}^{a_{2}} f_{1}\left(t, \sum_{i=-1}^{2^{(k-2)}-1} c_{i} \varphi_{k, i}(t), \sum_{i=-1}^{2^{(k-2)}-1} c_{i} \varphi_{k, i}^{\prime}(t)\right) d t \\
+\int_{a_{2}}^{a_{3}} f_{2}\left(t, \sum_{i=2^{(k-2)}-1}^{3 \cdot 2^{(k-2)}-1} c_{i} \varphi_{k, i}(t), \sum_{i=2^{(k-2)}-1}^{3 \cdot 2^{(k-2)}-1} c_{i} \varphi_{k, i}^{\prime}(t)\right) d t \\
+\int_{a_{3}}^{b} f_{3}\left(t, \sum_{i=3 \cdot 2^{(k-2)}-1}^{2^{k}-1} c_{i} \varphi_{k, i}(t), \sum_{i=3 \cdot 2^{(k-2)}-1}^{2^{k}-1} c_{i} \varphi_{k, i}^{\prime}(t)\right) d t
\end{gathered}
$$

Furthermore, we know that the support of uniform $d t h$-degree B-splines $B_{d}^{j}(t),(j=0, \pm 1, \cdots, \pm(d+1))$ are into the $[j, j+d+1]$ (Remark that hat functions is $\left.B_{1}^{0}=B_{1}\right)$. On the other hand, for all fix value of $t$, just $d$ consecutive terms of the sequence of $\left\{\cdots, B_{d}^{-1}(t)\right.$, $\left.B_{d}^{0}(t), B_{d}^{1}(t), \cdots\right\}$ is nonzero. Then, we have for $k \geq 2$ :

$$
\left\{\begin{array}{l}
\varphi_{k, i}(t) \equiv 0, \\
\forall t \in\left[a, a_{2}\right], 2^{(k-2)} \leq i \leq 2^{k}-1, \\
\varphi_{k, i}(t) \equiv 0, \\
\forall t \in\left[a_{2}, a_{3}\right], \\
-1 \leq i \leq 2^{(k-2)}-2 \text { and } 3 \cdot 2^{(k-2)} \leq i \leq 2^{k}-1, \\
\varphi_{k, i}(t) \equiv 0, \\
\forall t \in\left[a_{3}, b\right],-1 \leq i \leq 3 \cdot 2^{(k-2)}-2 .
\end{array}\right.
$$

So, from (13), we obtain

$$
\begin{aligned}
& \int_{a}^{b} f\left(t, u(t), u^{\prime}(t)\right) d t \\
= & \sum_{j=1}^{3} \int_{a_{j}}^{a_{j+1}} f_{j}\left(t, \sum_{i=-1}^{2^{k}-1} c_{i} \varphi_{k, i}(t), \sum_{i=-1}^{2^{k}-1} c_{i} \varphi_{k, i}^{\prime}(t)\right) d t \\
= & F\left(c_{-1}, c_{0}, c_{1}, \cdots, c_{2^{k}-1}\right)
\end{aligned}
$$

Therefore, in view of (14), the optimal problem
(10)-(11) reduces to the problem

$$
\min _{c_{-1}, c_{0}, c_{1}, \ldots, c_{2^{k}-1}} F\left(c_{-1}, c_{0}, c_{1}, \cdots, c_{2^{k}-1}\right)
$$

subject to

$$
\alpha_{1}=u(a)=c_{-1} .
$$

For finding $c_{0}, c_{1}, \ldots, c_{2^{k}-1}$, we have to solve the system $\frac{\partial F}{\partial c_{j}}=0,\left(j=0, \cdots, 2^{k}-1\right)$ Let $c_{-1}^{*}, c_{0}^{*}$, $c_{1}^{*}, \cdots, c_{2^{k}-1}^{*}$ be an approximate solution of (15)-(16) and set

$$
u_{k}^{*}(t)=\sum_{i=-1}^{2^{k}-1} c_{i}^{*} \varphi_{k, i}(t) .
$$

We assume integral operator $L$ define the following by the form

$$
L(u):=\int_{a}^{b} f\left(t, u(t), u^{\prime}(t)\right) d t .
$$

suppose that there exists a solution $u^{*} \in C([a, b])$ of (10) satisfying (11). (We assume $L\left(u^{*}\right) \neq-\infty$.) $\mathrm{M}$. Ahmadinia and G. B. Loghmani [21] shows that under reasonable conditions, $L\left(u_{k}^{*}\right)$ converges to $L\left(u^{*}\right)$ as $k \rightarrow+\infty$.

Theorem. Let $f$ in (12) have the property that for all $\varepsilon>0$ there exists $\delta>0$ such that $\mid f(t, u, v)-$ $f\left(t, u_{1}, v_{1}\right) \mid<\varepsilon$, whenever $\left|u-u_{1}\right|<\delta$ and $\left|v-v_{1}\right|<$ $\delta$. Let $u^{*} \in C([a, b])$ be a exact solution of the problem: (10)-(11). Assume $\left(u^{*}\right)^{\prime}$ is continuous on [ $a$, $b$ ]. The following assertions are true.

1) For all $\varepsilon>0$ there exists $k \geq 2$ and $h_{k}$ such that $0 \leq L\left(h_{k}\right)-L\left(u^{*}\right)<\varepsilon$, and $h_{k}$ satisfies (11).

2) Let $u_{k}^{*}$ be as in (17). Then $L\left(u^{*}\right) \leq L\left(u_{k}^{*}\right) \leq L\left(h_{k}\right)$ and $L\left(u_{k}^{*}\right) \rightarrow L\left(u^{*}\right)$ as $k \rightarrow+\infty$.

Proof. see [21].

The above theorem shows that for all $\varepsilon>0$ there exists an approximate solution $u_{k}^{*}$ for the optimal control problem (10)-(11) such that the difference between the value of $L\left(u_{k}^{*}\right)$ and the value $L\left(u^{*}\right)$ is at most $\varepsilon$.

Following the steps of the proof of above theorem we obtain the following corollary.

Corollary 1. All derivatives of the approximate solution converges to the related derivatives of the exact solution.

Remark 1. If the problem involves the higher derivative $u^{\prime \prime}$, we will use the uniform quadratic spline function $B_{2}$ in (9). Here, $B_{2}^{\prime \prime}$ is a left continuous step function. In this method, $B_{d}$ is used when the regularity of $B_{d}$ is minimal. That is, if the problem involves $u^{\prime}$ only, then $B_{d}^{\prime}(d=1)$ must be chosen to be a step function; if it involves $u^{\prime}$ and $u^{\prime \prime}$, then $B_{d}^{\prime \prime}(d=2)$ 
must be chosen to be a step function, etc.

\section{Applications}

To illustrate the application of the method developed in the previous sections, we suggested penalty functions technique for solving one dimension obstacle problems and deduction of existence and uniqueness solution of a systems (1)-(2). So, we consider the following second order obstacle boundary value problem about finding $u$ such that

$$
\begin{gathered}
\left\{\begin{array}{l}
-u^{\prime \prime}(t) \geq f(t), \quad \text { on } \Omega=[0, \pi] \\
u(t) \geq \psi(t), \quad \text { on } \Omega=[0, \pi]
\end{array}\right. \\
\left\{\begin{array}{l}
\left(u^{\prime \prime}-f(t)\right)(u(t)-\psi(t))=0, \quad \text { on } \Omega=[0, \pi] \\
u(0)=0 \text { and } u(\pi)=0,
\end{array}\right.
\end{gathered}
$$

where $f$ is a given continuous force acting on the beam and $\psi(t)$ is the elastic obstacle. Problem (18) describes the equilibrium configuration of an elastic beam, pulled at the ends and lying over an elastic obstacle. We study problem (18) in the framework of variational inequality approach. To do so, we define the set $K$ as

$$
\mathrm{K}=\left\{v \in H_{0}^{1}(\Omega): v \geq \psi \text { on } \Omega\right\} \neq \varnothing,
$$

which is a closed convex set in $H_{0}^{1}(\Omega)$, where $H_{0}^{1}(\Omega)$ is a Sobolev space, which is in fact a Hilbert space. For the definitions of the spaces $H_{0}^{1}(\Omega)$, see $[1,29]$. Here, $H_{0}^{1}(\Omega)$ can be the following form

$$
H_{0}^{1}(\Omega)=\left\{z \in H^{1}(\Omega): \lim _{t \rightarrow 0} Z(t)=0 \lim _{t \rightarrow \pi} Z(t)=0\right\}
$$

where $H^{1}(\Omega)$ is the space of absolutely continuous functions on the interval $[0, \pi]$ such that their first and second derivatives belonging to $L^{2}(\Omega)$. It can be easily shown that the energy functional associated with the obstacle problem (18), for all $v \in \mathrm{K}$ is

$$
\begin{aligned}
I[v] & =\int_{0}^{\pi}\left(\frac{d^{2} v}{d t^{2}}\right) v(t) d t-2 \int_{0}^{\pi} f(t) v(t) d t \\
& =a(v, v)-2<f, v>
\end{aligned}
$$

where

$$
a(u, v)=\int_{0}^{\pi}\left(\frac{d^{2} u}{d t^{2}}\right)\left(\frac{d^{2} v}{d t^{2}}\right) d t
$$

and

$$
<f, v>=\int_{0}^{\pi} f(t) v(t) d t
$$

Also it can be easily shown that $a(u, v)$ defined by (20) is bilinear, symmetric and positive (in fact, coercive $[5,7]$ ) and the functional $f$ defined by (21) is a linear continuous functional. It is well known $[1,5,7,29]$ that the minimum $u$ of the functional $I[v]$ defined by (19) on the closed convex set $\mathrm{K}$ in $H_{0}^{1}(\Omega)$ can be characterized by the variational inequality

$$
a(u, v-u) \geq<f, v-u>\text { for all } v \in \mathrm{K} .
$$

Thus, we conclude that the obstacle problem (18) is equivalent to solving the variational inequality problem (22). This equivalence has been used to study the existence of a unique solution of (18), [1,4,5]. Now using the idea of Lewy and Stampacchia [8], problem (22) can be written as

$$
\begin{gathered}
u^{\prime \prime}-\mu\{u-\psi\}(u-\psi)=f, 0<t<\pi, \\
u(0)=u(\pi)=0 .
\end{gathered}
$$

where $\psi$ is the obstacle function and $\mu(z)$ is a discontinuous function so that well known as the Penalty function defined by

$$
\mu(z)= \begin{cases}1, & z \geq 0, \\ 0, & z<0 .\end{cases}
$$

and $\psi$ is the given obstacle function defined by

$$
\psi(t)= \begin{cases}-1, & 0 \leq t \leq \frac{\pi}{4}, \\ 1, & \frac{\pi}{4} \leq t \leq \frac{3 \pi}{4}, \\ -1, & \frac{3 \pi}{4} \leq t \leq \pi .\end{cases}
$$

Equation (23) describes the equilibrium configuration of an obstacle string pulled at the ends and lying over elastic step of constant height 1 and unit rigidity. Sinec obstacle function $\psi$ is known, so it is possible to solve the problem in the interval $[0, \pi]$. From Equations (23)-(24), we obtain the following system of differential equations:

$$
u^{\prime \prime}=\left\{\begin{array}{cr}
f, & 0 \leq t \leq \frac{\pi}{4} \text { and } \frac{3 \pi}{4} \leq t \leq \pi, \\
u+f-1, & \frac{\pi}{4} \leq t \leq \frac{3 \pi}{4},
\end{array}\right.
$$

with the boundary conditions

$$
u(0)=u(\pi)=0,
$$

and the condition of continuity of $u$ and $u^{\prime}$ at $t=\frac{\pi}{4}$ and $\frac{3 \pi}{4}$.

\section{Numerical Results and Discussion}

In this section, we consider second linear and nonlinear problems will be tested by using the method discussed above. The B-spline solutions of Equations (1)-(2) was 
obtained using a linear combination $u_{k}^{*}(t)=\sum_{i=-2}^{3 \cdot 2^{k-1}-1}$ $c_{i}^{*} \Phi_{k, i}(t)$, and the minimization problem was solved by Maple 12. The least square errors (LSE) in the analytical solutions for test problem 1, 2 and 3 were calculated and are depicted in Tables 1-3.

Test problem 1 ([3,17-19], Example 1).

Consider the linear system of differential Equations (25)-(26) when $f(t)=0$, takes the following form:

$$
u^{\prime \prime}= \begin{cases}0, & 0 \leq t \leq \frac{\pi}{4} \\ u-1, & \frac{\pi}{4} \leq t \leq \frac{3 \pi}{4},\end{cases}
$$

with boundary condition (26). The analytical solution for this problem is given by

$$
\begin{gathered}
u(t)= \begin{cases}\frac{4 t}{\gamma_{1}}, & 0 \leq t \leq \frac{\pi}{4},\end{cases} \\
u(t)=\left\{\begin{array}{c}
1-\frac{4 \cosh \left(\frac{\pi}{2}-t\right)}{\gamma_{2}}, \quad \frac{\pi}{4} \leq t \leq \frac{3 \pi}{4}, \\
\frac{4(\pi-t)}{\gamma_{1}}, \quad \frac{3 \pi}{4} \leq t \leq \pi,
\end{array}\right.
\end{gathered}
$$

where $\gamma_{1}:=\pi+4 \operatorname{coth}\left(\frac{\pi}{4}\right)$ and $\gamma_{2}:=\gamma_{1} \sinh \left(\frac{\pi}{4}\right)$.

The problem (27) was solved using the mehtod described in Section 2 and 3 with a variety of $h$ values with respect to $k \geq 3$. The observed least square errors (LSE) are depicted in Table 1. We use the method and obtain this results;

Approximate solution $u_{k}^{*}$ for $k=3$ :

$$
\begin{aligned}
& u_{3}^{*}(t)=-0.05662 \Phi_{3,-2}(t)+0.05662 \Phi_{3,-1}(t) \\
& +0.16985 \Phi_{3,0}(t)+0.28308 \Phi_{3,1}(t)+0.39629 \Phi_{3,2}(t) \\
& +0.46765 \Phi_{3,3}(t)+0.50213 \Phi_{3,4}(t)+0.50213 \Phi_{3,5}(t) \\
& +0.46765 \Phi_{3,6}(t)+0.39629 \Phi_{3,7}(t)+0.28308 \Phi_{3,8}(t) \\
& +0.16985 \Phi_{3,9}(t)+(0.56617 e-1) \Phi_{3,10}(t) \\
& +(-0.56617 e-1) \Phi_{3,11}(t)
\end{aligned}
$$

Approximate solution $u_{k}^{*}$ for $k=4$ :

$$
\begin{aligned}
& u_{4}^{*}(t)=-0.02832 \Phi_{4,-2}(t)+0.02832 \Phi_{4,-1}(t) \\
& +(0.84974 e-1) \Phi_{4,0}(t)+0.14162 \Phi_{4,1}(t) \\
& +0.19827 \Phi_{4,2}(t)+0.25492 \Phi_{4,3}(t)+0.31157 \Phi_{4,4}(t) \\
& +0.36821 \Phi_{4,5}(t)+0.41400 \Phi_{4,6}(t)+0.44972 \Phi_{4,7}(t) \\
& +0.47599 \Phi_{4,8}(t)+0.49325 \Phi_{4,9}(t)+0.50181 \Phi_{4,10}(t) \\
& +0.50181 \Phi_{4,11}(t)+0.49325 \Phi_{4,12}(t)+0.47599 \Phi_{4,13}(t)
\end{aligned}
$$

Table 1. Least square error (LSE) for Test problem 1.

\begin{tabular}{|c|c|c|c|c|}
\hline$\left(u_{k}^{*}\right)^{(j)}$ & LES $(k=3)$ & LES $(k=4)$ & LES $(k=5)$ & LES $(k=6)$ \\
\hline$j=0$ & $1.6575 e-7$ & $1.6575 e-7$ & $5.6080 e-10$ & $1.1997 e-10$ \\
\hline$j=1$ & $2.4876 e-6$ & $1.5748 e-7$ & $9.8946 e-9$ & $8.4401 e-10$ \\
\hline$j=2$ & $5.0502 e-4$ & $1.2968 e-4$ & $3.2325 e-5$ & $8.0904 e-6$ \\
\hline
\end{tabular}

$+0.44972 \Phi_{4,14}(t)+0.41400 \Phi_{4,15}(t)+0.31157 \Phi_{4,16}(t)$

$+0.25492 \Phi_{4,17}(t)+0.19827 \Phi_{4,18}(t)+0.14162 \Phi_{4,19}(t)$

$+0.36821 \Phi_{4,20}(t)+(0.84974 e-1) \Phi_{4,21}(t)$

$+(0.28325 e-1) \Phi_{3,22}(t)+(-0.28325 e-1) \Phi_{3,23}(t)$

The approximate solution can also be obtained for $k=5,6$.

We would like to emphasize that the present method has the advantage of the ability of approximating the derivatives of $u$ and $u^{\prime}$ on $[a, b]$ where as other parametric spline and finite difference methods $[2,17]$ do not have this ability. Mach as, cubic spline method [3] has the ability of approximating the first derivative, but it hasn't the ability of approximating the second derivatives such that the our method can be approximating both of first and second derivatives.

\section{Test problem 2.}

Consider the system of differential Equations (25)-(26) when $f(t)=2$, takes the following form:

$$
u^{\prime \prime}= \begin{cases}2, & 0 \leq t \leq \frac{\pi}{4} \text { and } \frac{3 \pi}{4} \leq t \leq \pi, \\ u+1, & \frac{\pi}{4} \leq t \leq \frac{3 \pi}{4},\end{cases}
$$

with boundary condition (25). The analytical solution for this problem is given by

$$
u(t)=\left\{\begin{array}{c}
t^{2}+\left(\frac{\left(\pi^{2}-16\right) \sinh \left(\frac{\pi}{4}\right)}{4 \gamma}-\frac{\pi}{2}\right) t, \\
0 \leq t \leq \frac{\pi}{4}, \\
-1-\frac{\left(\pi^{2}-16\right)}{4 \gamma} \cosh \left(\frac{\pi}{2}-t\right), \\
\frac{\pi}{4} \leq t \leq \frac{3 \pi}{4}, \\
t^{2}+\left(\frac{\left(\pi^{2}-16\right) \sinh \left(\frac{\pi}{4}\right)}{4 \gamma}-\frac{\pi}{2}\right)(\pi-t) \\
+\frac{\pi}{2}(\pi-3 t),
\end{array}\right)
$$




$$
u(t)=\left\{\frac{3 \pi}{4} \leq t \leq \pi,\right.
$$

where $\gamma:=\pi \sinh \left(\frac{\pi}{4}\right)+4 \cosh \left(\frac{\pi}{4}\right)$.

The problem (29) was solved using the mehtod described in Section 2 and 3 with a variety of $h$ values with respect to $k \geq 3$. The observed least square errors (LSE) are depicted in Table 2. We use the method and obtain this results;

Approximate solution $u_{k}^{*}$ for $k=3$ :

$$
\begin{aligned}
& u_{3}^{*}(t)=+0.22731 \Phi_{3,-2}(t)-0.22731 \Phi_{3,-1}(t) \\
& -0.54485 \Phi_{3,0}(t)-0.72531 \Phi_{3,1}(t)-0.76869 \Phi_{3,2}(t) \\
& -0.79603 \Phi_{3,3}(t)-0.80924 \Phi_{3,4}(t)-0.80924 \Phi_{3,5}(t) \\
& -0.79603 \Phi_{3,6}(t)-0.76869 \Phi_{3,7}(t)-0.72531 \Phi_{3,8}(t) \\
& -0.54485 \Phi_{3,9}(t)-0.22731 \Phi_{3,10}(t)+0.22731 \Phi_{3,11}(t)
\end{aligned}
$$

The approximate solution can also be obtained for $k=4,5,6$.

\section{Test problem 3 ([29], Example).}

Consider the system of differential equation:

$$
u^{\prime \prime \prime}=\left\{\begin{array}{cc}
0, & 0 \leq t \leq \frac{1}{4} \\
u-1, & \frac{1}{4} \leq t \leq \frac{3}{4} \\
0, & \frac{3}{4} \leq t \leq 1
\end{array}\right.
$$

with boundary conditions,

$$
u(0)=u^{\prime}(0)=u^{\prime}(1)=0 \text {. }
$$

The analytical solution for this problem is given by

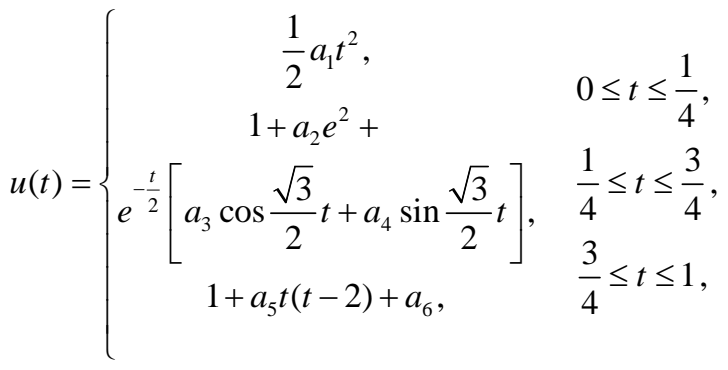

We can find the constants $a_{i}, i=1,2, \cdots, 6$ by solving a system of linear equations constructed by applying the conitinuity conditions of $u, u^{\prime}, u^{\prime \prime}$ at $t=\frac{1}{4}$ and $t=\frac{3}{4}$.

We consider $a_{i}=0.24391, \quad a_{i}=-0.17847, \quad a_{i}=$ $-0.81893, a_{i}=-0.30266, a_{i}=-0.24213, a_{i}=$ -0.65376 .

The homogeneous linear problem (31) was solved
Table 2. Least square error (LSE) for test problem 2.

\begin{tabular}{|c|c|c|c|c|}
\hline$\left(u_{k}^{*}\right)^{(j)}$ & LES $(k=3)$ & LES $(k=4)$ & LES $(k=5)$ & LES $(k=6)$ \\
\hline$j=0$ & $2.4377 e-8$ & $1.5765 e-9$ & $4.9304 e-1$ & $1.3667 e-10$ \\
\hline$j=1$ & $3.6519 e-7$ & $2.3133 e-8$ & $1.5552 e-9$ & $6.3775 e-10$ \\
\hline$j=2$ & $7.4138 e-5$ & $1.8891 e-5$ & $4.7577 e-6$ & $1.1897 e-6$ \\
\hline
\end{tabular}

Table 3. Least square error (LSE) for test problem 3.

\begin{tabular}{c|c|c|c|c|}
\hline$\left(u_{k}^{*}\right)^{(j)}$ & LES $(k=3)$ & LES $(k=4)$ & LES $(k=5)$ & LES $(k=6)$ \\
\hline$j=0$ & $3.3013 e-13$ & $2.0636 e-14$ & $1.2931 e-15$ & $7.9937 e-17$ \\
\hline$j=1$ & $4.0337 e-12$ & $2.5158 e-13$ & $1.5714 e-14$ & $9.8027 e-16$ \\
\hline$j=2$ & $2.4877 e-10$ & $1.5545 e-11$ & $9.7153 e-13$ & $6.0670 e-14$ \\
$j=3$ & $1.0822 e-6$ & $2.7041 e-7$ & $6.7593 e-8$ & $1.6897 e-8$ \\
\hline
\end{tabular}

using the mehtod described in Section 2 and 3 with a variety of $h$ values with respect to $k \geq 3$. The observed least square errors (LSE) are given in Table $\mathbf{3}$. We use the method and obtain this results.

\section{Conclusions}

The computations associated with the three examples discussed above were performed by using Maple 12 . Comparing the obtained results with other works $[3,13$, 16-19], this method was clearly reliable if compared with grid points techniques where solution is defined at grid points only. Moreover the method yields a good result even for small $k$.

\section{Acknowledgements}

The authors are grateful to the anonymous reviewers for their helpful comments, which greatly improves this paper.

\section{References}

[1] N. Kikuchi and J. T. Oden, "Contact Problems in Elasticity,” SIAM Publishing Company, Philadelphia, 1988.

[2] M. A. Noor and S. I. A. Tirmizi, "Finite Difference Techniques for Solving Obstacle Problems,” Applied Mathematics Letters, Vol. 1, No. 3, 1988, pp. 267-271. doi:10.1016/0893-9659(88)90090-0

[3] E. A. Al-Said, " The Use of Cubic Splines in the Numerical Solution of a System of Second Order Boundary Value Problems," Computers \& Mathematics with Applications, Vol. 42, No. 6, September 2001, pp. 861-869. doi:10.1016/S0898-1221(01)00204-8

[4] C. Baiocchi and A. Capelo, "Variational and Quasi-Variational Inequalities,” John Willey and Sons, New York, 1984.

[5] A. Friedman, "Variational Principles and Free-Boundary Problems,” John Willey and Sons, New York, 1982. 
[6] R. Glowinski, J. L. Lions and R. Tremolieres, "Numerical Analysis of Variational Inequalities,” North-Holland Pub., Amesterdam, 1981.

[7] D. Kinderlehrer and G. Stampacchia, "An Introduction to Variational Inequalities and Their Applications,” New York Academic Press, New York, 1980.

[8] H. Lewy and G. Stampacchia, "On the Regularity of the Solutions of the Variational Inequalities," Communications on Pure and Applied Mathematics, Vol. 22, No. 2, March 1969, pp. 153-188.

[9] J. L. Lions and G. Stampacchia, "Variational Inequalities," Communications on Pure and Applied Mathematics, Vol. 20, No. 3, August 1967, pp. 493-519. doi:10.1002/cpa.3160200302

[10] J. F. Rodrigues, "Obstacle Problems in Mathematical Physics,” North-Holland Pub., Amesterdam, 1987.

[11] F. Villaggio, "The Ritz Method in Solving Unilateral Problems in Elasticity,” Meccanica, Vol. 16, No. 3, 1981, pp. 123-127. doi:10.1007/BF02128440

[12] M. A. Noor and A. K. Khalifa, "Cubic Splines Collocation Methods for Unilateral Problems," International Journal of Engineering Science, Vol. 25, No. 11, 1987, pp. 1527-1530. doi:10.1016/0020-7225(87)90030-9

[13] E. A. Al-Said, "Smooth Spline Solutions for a System of Second Order Boundary Value Problems," Jounal of Natural Geometry, Vol. 16, No. 1, 1999, pp. 19-28.

[14] E. A. Al-Said, M. A. Noor and A. A. Al-Shejari, "Numerical Solutions for System of Second Order Boundary Value Problems,” The Korean Journal of Computational \& Applied Mathematics, Vol. 5,No. 3, 1998, pp. 659-667.

[15] E. A. Al-Said, "Spline Solutions for System of Second Order Boundary Value Problems,” International Journal of Computer Mathematics, Vol. 62, No. 1, 1996, pp. 143154. doi:10.1080/00207169608804531

[16] E. A. Al-Said, "Spline Methods for Solving System of Second Order Boundary Value Problems," International Journal of Computer Mathematics, Vol. 70, No. 4, 1999, pp. 717-727. doi:10.1080/00207169908804784

[17] A. Khan and T. Aziz, “ Parametric Cubic Spline Approach to the Solution of a System of Second Order Boundary Value Problems," Journal of Optimization Theory and Applications, Vol. 118, No. 1, July 2003, pp. 4554. doi:10.1023/A:1024783323624

[18] Siraj-ul-Islam, M. A. Noor, I. A. Tirmizi and M. A. Khan, "Quadratic Non-Polynomial Spline Approach to the
Solution of a System of Second-Order Boundary-Value Problems," Applied Mathematics and Computation, Vol. 179, No. 1, August 2006, pp. 153-160. doi:10.1016/j.amc.2005.11.091

[19] Siraj-ul-Islam and I. A. Tirmizi, "Non-Polynomial Spline Approach to the Solution of a System of Second-Order Boundary-Value Problems," Applied Mathematics and Computation, Vol. 173,No. 2. February 2006, pp. 12081218. doi:10.1016/j.amc.2005.04.064

[20] J. H. Ahlberg, E. N. Nilson and J. L. Walsh, "The Theory of Splines and Their Applications,” Academic Press, New York, 1967.

[21] C. De Boor, “A Practical Guide to Splines,” SpringerVerlag, New York, 1978.

[22] M. Ahmadinia and G. B. Loghmani, "Splines and AntiPeriodic Boundary Value Problems," International Journal of Computer Mathematics, Vol. 84, No. 12, December 2007, pp. 1843-1850. doi:10.1080/00207160701336466

[23] I. Daubecheis, "Orthonormal Bases of Compactly Supported Wavelets," Communications on Pure and Applied Mathematics, Vol. 41, No. 7, October 1988, pp. 909-996.

[24] G. B. Loghmani and M. Ahmadinia, "Numerical Solution of Sixth-Order Boundary Value Problems with Sixth-Degree B-Spline Functions,” Applied Mathematics and Computation, Vol. 186, No. 2, March 2007, pp. 992-999. doi:10.1016/j.amc.2006.08.068

[25] G. B. Loghmani and M. Ahmadinia, "Numerical Solution of Third-Order Boundary Value Problems," Iranian Journal of Science \& Technology, Vol. 30, No. A3, 2006, pp. 291-295.

[26] M. Radjabalipour, "Application of Wavelet to Optimal Control Problems,” International Conference on Scientific Computation and Differential Equations, Vancouver, July 29-August 3, 2001.

[27] L. L. Schumaker, "Spline Functions: Basic Theory,” John Willey and Sons, New York, 1981.

[28] J. Stoer and R. Bulirsch, "Introduction to Numerical Analysis,” Springer-Verlag, Berlin 1993.

[29] E. A. Al-Said, M. A. Noor and A. A. Al-Shejari, "Numerical Solutions of Third-Order System of Boundary Value Problems," Applied Mathematics and Computation, Vol. 190, No. 1, July 2007, pp. 332-338. doi:10.1016/j.amc.2007.01.031 